\title{
A Likely Biogenetic Gateway Linking 2-Aminoimidazolinones Metabolites of Sponges to Proline: Spontaneous Oxidative Conversion of the Pyrrole-Proline-Guanidine Pseudo-peptide to Dispacamide A.
}

\author{
Nathalie Travert and Ali Al-Mourabit* \\ Institut de Chimie des Substances Naturelles du CNRS, BP \&, 91198 Gif-sur-Yvette, France
}

\section{Experimental section}

General: chemicals were purchased from commercial sources and used without further treatment except when specified. THF was freshly distilled from sodium benzophenone under nitrogen. Methylene chloride and triethyamine were distilled from calcium hydride. Flash chromatography was carried out using Merck silica gel 40-60 (230-400 mesh).Meltipg points were determined with a Buchi-B540 and are uncorrected. $\mathrm{H}$ NMR spectra were recorded at Bruker 250, 300 and $400 \mathrm{MHz}, \mathrm{C}$ NMR spectra at 62.5 , 75 and $100 \mathrm{MHz}$ with tetramethylsilane or solvent $\left(\mathrm{CDCl}_{3}, \mathrm{CD}_{3} \mathrm{OD}, \mathrm{DMSOd}_{6}\right)$ as internal standard $(\mathrm{d} \mathrm{ppm})$. ${ }^{1} \mathrm{H}$ and ${ }^{3} \mathrm{C}$ were assigned by means of COSY, HMBC, HMQC and and NOESY spectra. FT-IR spectra were recorded on a Perking Elmer-BX spectrumeter using $\mathrm{CHCl}_{3}$ or $\mathrm{KBr}$ techniques.

1-(1H -Pyrrole-2-carbonyl)-pyrrolidine-2-carboxylic acid methyl ester (10):

To a mixture of 2-pyrrolecarboxylic acid (8) (2.00 g, $18.02 \mathrm{mmol}$, 1equiv.), L-proline methyl ester (9) (11.90 g, $72.07 \mathrm{mmol} ., 4$ equiv.) and DMAP (catalytic) in anhydrous dichloromethane $(60 \mathrm{~mL})$ was added under argon at $0^{\circ} \mathrm{C}$ the 1 -(3-dimethylaminopropyl)3-ethylcarbodiimide $\left(6.90 \mathrm{~g} ; 36.04 \mathrm{mmol}, 2\right.$ equiv.). After $30 \mathrm{~min}$ stirring at $0^{\circ} \mathrm{C}$ and $2 \mathrm{~h}$ at room temperature, the reaction mixture was washed with water, dried with $\mathrm{MgSO}_{4}$, and then dried under vacuum. Silica-gel column chromatography with heptane/EtOAc : $1 / 1$ gave $10(3.80 \mathrm{~g})$ in $95 \%$ yield.

10

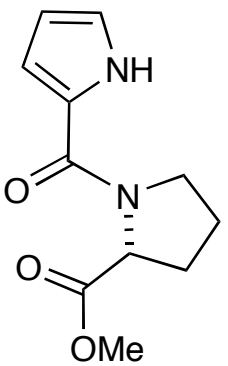

10: white powder; $\mathrm{mp}=71-7{ }^{\circ} \mathrm{C}$; MS-EI: $222.0[\mathrm{M}]^{+}, 163.0\left[\mathrm{M}-\mathrm{CO}_{2} \mathrm{Me}\right], \mathrm{HRMS}$ calcd. for $\mathrm{C}_{11} \mathrm{H}_{14} \mathrm{~N}_{2} \mathrm{O}_{3}: 245,0902[\mathrm{M}+\mathrm{Na}]^{+}$; found: 245,0882 [M+ Na] ${ }^{+} ;{ }^{1} \mathrm{H}$ NMR $\left(300 \mathrm{MHz}, \mathrm{CDCl}_{3}\right) \delta 10.23(\mathrm{bs}, 1 \mathrm{H}), 6.97(\mathrm{~m}, 1 \mathrm{H}), 6.65(\mathrm{~m}, 1 \mathrm{H}), 6.28(\mathrm{~m}, 1 \mathrm{H}), 4.66(\mathrm{~m}, 1 \mathrm{H}), 3.93$ $(\mathrm{m}, 2 \mathrm{H}), 3.75(\mathrm{~s}, 3 \mathrm{H}), 2.21(\mathrm{~m}, 2 \mathrm{H}), 2,05(\mathrm{~m}, 2 \mathrm{H}) ;{ }^{13} \mathrm{C} \mathrm{NMR}\left(75 \mathrm{MHz}, \mathrm{CDCl}_{3}\right) \delta 173.1,160.7,125.3,121.9,112.8,110.0,60.2$, 52.3, 48.4, 28.8, $25.5 \mathrm{ppm}$; IR $\left(\mathrm{NaCl}, \mathrm{n} \mathrm{cm}^{-1}\right): 3247,1743,1586,[\alpha]_{\mathrm{D}}{ }^{23}-43.3(\mathrm{c} 1.28, \mathrm{MeOH})$.

1,2,3,10a-tetrahydro-dipyrrolo[1,2-a; 1',2'-d]pyrazine-5,10-dione (11):

Methyl ester $10(710 \mathrm{mg}, 3.20 \mathrm{mmol})$ was dissolved in THF $(20 \mathrm{~mL})$ under argon and was cooled to $0^{\circ} \mathrm{C}$. Sodium hydride $(121 \mathrm{mg}$, $4.80 \mathrm{mmol}$ ) was added and the mixture was stirred for one hour. The mixture was then poured into phosphate buffer $\mathrm{pH} 7(30 \mathrm{~mL})$ and the products were extracted with EtOAc. After removal of the solvents under reduced pressure, the residue was purified on silica gel column chromatography using Heptane/EtOAc: 1/4 as eluent to give $\mathbf{1 1}$ as a white solid (448 $\mathrm{mg}, 2.37 \mathrm{mmol}, 74 \%$ yield).

11<smiles>O=C1c2cccn2C(=O)N2CCCC12</smiles>

11: White solid; $\mathrm{mp}=135{ }^{\circ} \mathrm{C}$; HRMS (ESI) calcd. for $\mathrm{C}_{10} \mathrm{H}_{1} \mathrm{~N}_{2} \mathrm{O}_{2} 191.0821[\mathrm{M}+\mathrm{H}]^{+}$, found: $191.0814[\mathrm{M}+\mathrm{H}]^{+}$; ${ }^{1} \mathrm{H} \mathrm{NMR}(300 \mathrm{MHz}$, $\left.\mathrm{CDCl}_{3}\right) \delta 1.97-2.22(\mathrm{~m}, 3 \mathrm{H}), 2.51-2.61(\mathrm{~m}, 1 \mathrm{H}), 3.59-3.70(\mathrm{~m}, 1 \mathrm{H}), 3.79-3.90(\mathrm{~m}, 1 \mathrm{H}), 4.49(\mathrm{dd}, J=9.5,6.2 \mathrm{~Hz}, 1 \mathrm{H}), 6.48(\mathrm{dd}, J=$ 3.3, $\left.3.3 \mathrm{~Hz}, 1 \mathrm{H}), 7.07(\mathrm{dd}, J=3.3,1.8 \mathrm{~Hz}, 1 \mathrm{H}), 7.45(\mathrm{dd}, J=3.3,1.8 \mathrm{~Hz}, 1 \mathrm{H}) ;{ }^{13} \mathrm{C} \mathrm{NMR}_{(\mathrm{CDCl}}, 75 \mathrm{MHz}\right) \delta 22.6,29.3,45.0,61.8$, 115.8, 118.1, 119.1, 127.8, 155.5, 164.8; MS-EI $190.0\left(\mathrm{M}^{+}, 100\right), 162.0(\mathrm{M}-(\mathrm{CO}), 30), 134.0(\mathrm{M}-2(\mathrm{CO}), 80 . \mathrm{IR}(\mathrm{NaCl}, \mathrm{n} \mathrm{max} \mathrm{cm}$ 1) $1727,1641,1435,1409$. 
N-[1-( $1 H$-pyrrole-2carbonyl)-pyrrolidine-2-carbonyl]-guanidine (6) and 4-hydroxy-5-oxo-4-\{3-[(1Hpyrrole-2-carbonyl)-amino]-propyl\}-imidazolin-2-ylidene (12) from 11 :

A solution of tricycle $11(0.20 \mathrm{~g}, 1.05 \mathrm{mmol} ., 1$ equiv.) in THF $(10 \mathrm{~mL})$ was added to a stirred mixture of guanidine hydrochloride (0.12 g, 1.26 mmol., 1.2 equiv.) in THF $(6 \mathrm{~mL})$ and $10 \mathrm{M} \mathrm{NaOH}(0.13 \mathrm{~mL}, 1.26 \mathrm{mmol}$., 1.2 equiv.). The reaction mixture was stirred for $1 \mathrm{~h}$ at room temperature and dried under vacuum. Flash chromatography on silica gel using AcOEt/MeOH : 2 / 1 saturated with ammonia afforded $0.13 \mathrm{~g} \mathrm{(42 \% )}$ of $\mathbf{6}$ and $0.17 \mathrm{~g} \mathrm{(44 \% )}$ of $\mathbf{1 2}$.<smiles>N=C(N)NC(=O)C1CCCN1C(=O)c1ccc[nH]1</smiles><smiles>NC1=NC(O)(CCCNC(=O)c2ccc[nH]2)C(=O)N1</smiles>

(6 rotameres): NMR ${ }^{1} \mathrm{H}$ (DMSO-d6, $\left.300 \mathrm{MHz}\right) \delta 12.24$ and $11.40(1 \mathrm{H}, 2 \mathrm{bs}), 6.89$ and $6.80(1 \mathrm{H}, 2 \mathrm{bs}), 6.62$ and $6.53(1 \mathrm{H}, 2 \mathrm{bs})$, 6.14 et $6.04(1 \mathrm{H}, 2 \mathrm{bs}), 4.40-4.37(1 \mathrm{H}, \mathrm{m}), 3.78-3.44(2 \mathrm{H}, \mathrm{m}), 2.10-1.89(4 \mathrm{H}, \mathrm{m})$.

12: amorphous solid, ${ }^{1} \mathrm{H}$ RMN (DMSO-d6, $\left.300 \mathrm{MHz}\right) \delta 11.41(1 \mathrm{H}, \mathrm{bs}), 8.03(1 \mathrm{H}, \mathrm{t}, J=6,0 \mathrm{~Hz}), 7.50-7.14(3 \mathrm{H}, \mathrm{m}), 6.81(1 \mathrm{H}$, $\mathrm{m}), 6.73(1 \mathrm{H}, \mathrm{m}), 6.05(1 \mathrm{H}, \mathrm{m}), 3.19-3.14(2 \mathrm{H}, \mathrm{m}), 1.72-1.21(4 \mathrm{H}, \mathrm{m}) ;{ }^{13} \mathrm{C}$ NMR (DMSO-d6, 75 MHz) $\delta 174.2,160.1,159.6$, $128.8,128.1,125.4$ et $125.2,112.1,111.3,108.8,108.5,64.8,60.2,48.0,28.3,24.8$.

\section{Preparation of $12, \mathrm{CF}_{3} \mathrm{CO}_{2} \mathrm{H}$ from 11 and Boc-guanidine:}

To a dichloromethane $(40 \mathrm{~mL})$ solution of tricycle $11(0.60 \mathrm{~g}, 3.16 \mathrm{mmol}$. ) was added N-Boc guanidine (1,0 g, $6.32 \mathrm{mmol} ., 2$ equiv.) and stirred overnight at room temperature. The reaction mixture was evaporated and the residue purified by silica gel column flash chromatography using $\mathrm{CH}_{2} \mathrm{Cl}_{2} / \mathrm{MeOH}$ : 95/5. Regioisomers $\mathbf{1 3 + 1 4}(0.48 \mathrm{~g})$ were obtained as a balanced mixture in $42 \%$ yield. The mixture was deprotected to $\mathbf{1 2}$ as below:

To a dichloromethane $(4 \mathrm{~mL})$ suspension of the unsparable $\mathbf{1 3}+\mathbf{1 4}(0.15 \mathrm{~g}, 0.4 \mathrm{mmol}$.), was added trifluroacetic acid $(3 \mathrm{~mL})$ at room temperature and stirred for 2 hours. The reaction mixture was concentrated and dried. $0.16 \mathrm{~g}$ of compound 12 was obtained quantitatively.<smiles>[R4]C(=O)OCCCC1(O)N=C(N)NC1=O</smiles>

12, $\mathbf{C ~ F}_{3} \mathbf{C O}_{2} \mathbf{H}$ : Amorphous solid, ESI-MS: $288.2[\mathrm{M}+\mathrm{Na}]^{+}, 266.2[\mathrm{M}+\mathrm{H}]^{+}, 248.1\left[\mathrm{M}-\mathrm{H}_{2} \mathrm{O}\right] ; \mathrm{HRMS}$ calcd. for $\mathrm{C}_{11} \mathrm{H}_{15} \mathrm{~N}_{5} \mathrm{O}_{3}: 288,1073$ $[\mathrm{M}+\mathrm{Na}]^{+}$, found: $288,1083[\mathrm{M}+\mathrm{Na}]^{+} ;{ }^{1} \mathrm{H}$ NMR (DMSO- $\left.d 6,300 \mathrm{MHz}\right) \delta 11.37(1 \mathrm{H}, \mathrm{bs}), 10.28(1 \mathrm{H}, \mathrm{bs}), 9.21(2 \mathrm{H}, \mathrm{bs}), 8.02(1 \mathrm{H}, \mathrm{t}, J$ $=5.6 \mathrm{~Hz}), 7.17(1 \mathrm{H}, \mathrm{s}), 6.82(1 \mathrm{H}, \mathrm{m}), 6.72(1 \mathrm{H}, \mathrm{m}), 6.05(1 \mathrm{H}, \mathrm{m}), 3.16(2 \mathrm{H}, \mathrm{m}), 1.80(2 \mathrm{H}, \mathrm{m}), 1.50$ and $1,40(2 \mathrm{H}, \mathrm{m}) ;{ }^{13} \mathrm{C} \mathrm{NMR}$ (DMSO-d6, $75 \mathrm{MHz}) \delta 160.9,150.5,126.7,121.4,110.0,108.8,86.1,78.7,38.5,33.5,28.2,24.1$.

Indicative data for the balanced mixture of $\mathbf{1 3}$ and $\mathbf{1 4}$ :

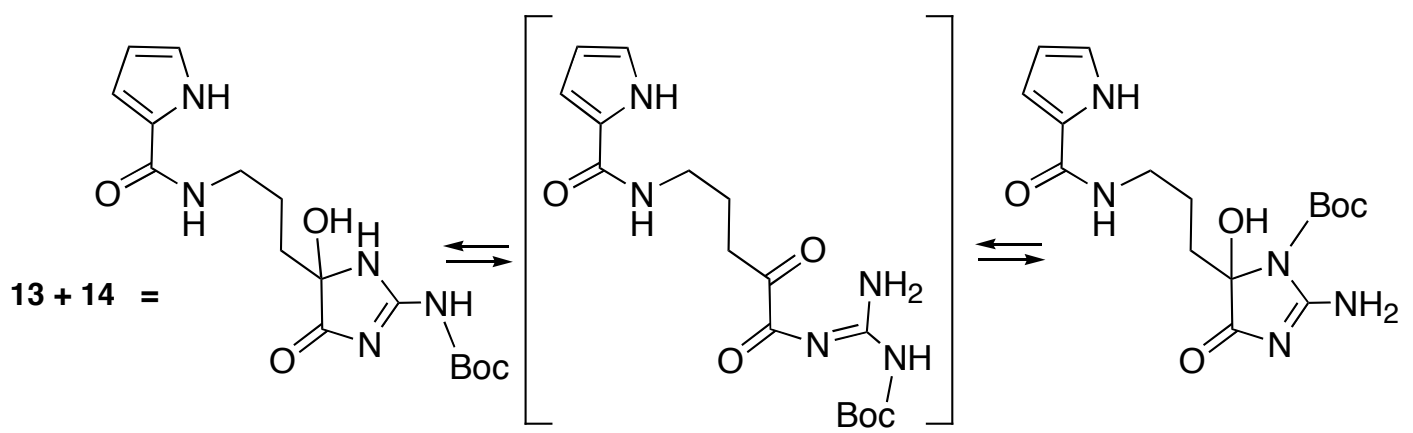

White solid, $\mathrm{mp}=172^{\circ} \mathrm{C}$, ESI-MS: $404.0[\mathrm{M}+\mathrm{K}]^{+}, 388.0[\mathrm{M}+\mathrm{Na}]^{+}, 366.1[\mathrm{M}+\mathrm{H}]^{+}, 348.1\left[\mathrm{M}-\mathrm{H}_{2} \mathrm{O}\right], 266.1[\mathrm{M}-\mathrm{Boc}]$, HRMS calcd. for $\mathrm{C}_{16} \mathrm{H}_{23} \mathrm{~N}_{5} \mathrm{O}_{5}: 388,1597[\mathrm{M}+\mathrm{Na}]^{+}$, found: 388,1575 [M+Na] ${ }^{+} .{ }^{1} \mathrm{H}$ NMR (DMSO-d6, $\left.300 \mathrm{MHz}\right) \delta 11.38(1 \mathrm{H}, \mathrm{bs}), 11.10(1 \mathrm{H}, \mathrm{bs}), 9.04$ $(1 \mathrm{H}, \mathrm{bs}), 7.98(1 \mathrm{H}, \mathrm{t}, J=5.6 \mathrm{~Hz}), 6.82(1 \mathrm{H}, \mathrm{m}), 6.72(1 \mathrm{H}, \mathrm{m}) .6 .67(1 \mathrm{H}, \mathrm{s}), 6.05(1 \mathrm{H}, \mathrm{m}), 3.16(2 \mathrm{H}, \mathrm{m}), 1.91-1.69(2 \mathrm{H}, \mathrm{m}), 1.41$ 
$(9 \mathrm{H}, \mathrm{s}), 1.34-1.20(2 \mathrm{H}, \mathrm{m}) ;{ }^{13} \mathrm{C}$ NMR (DMSO-d6, $\left.75 \mathrm{MHz}\right) \delta 160.9,150.5,126.7,121.4,110.0,108.8,86.1,78.7,38.5,33.5$, 28.2, 24.1; IR ( $\left.\mathrm{NaCl}, \mathrm{n} \max \mathrm{cm}^{-1}\right): 1742,1626,1564$.

\section{Preparation of $13+14$ from 10 and Boc-guanidine:}

To a THF ( $5 \mathrm{~mL})$ solution of methyl ester 1 (220 mg, $1 \mathrm{mmol})$, was added N-Boc guanidine (30 mg, 2 mmol., 2 equiv.) and stirred $3 \mathrm{~h}$ at reflux. The reaction mixture was evaporated and the residue purified by silica gel column flash chromatography using $\mathrm{CH}_{2} \mathrm{Cl}_{2} / \mathrm{MeOH}$ : 95/5. Regioisomers $\mathbf{1 3 + 1 4}$ and 15 were obtained in $46 \%$ and $7 \%$ yield respectively.

15<smiles>O=C1CCCN1C(=O)c1ccc[nH]1</smiles>

\section{1 -(1H-Pyrrole-2-carbonyl)-pyrrolidin-2-one 15:}

White solid, EI-MS: $178.0[\mathrm{M}]^{+}, 94.0\left[\mathrm{M}\right.$-pyrrolidinone ${ }^{+}$, 66.0. ${ }^{1} \mathrm{H} \mathrm{NMR}\left(\mathrm{CDCl}_{3}, 300 \mathrm{MHz}\right) \delta 11.58(1 \mathrm{H}, \mathrm{bs}), 7.20(1 \mathrm{H}, \mathrm{m}), 6.94$ $(1 \mathrm{H}, \mathrm{m}), 6.24(1 \mathrm{H}, \mathrm{m}), 6.82(1 \mathrm{H}, \mathrm{m}), 3.97(1 \mathrm{H}, \mathrm{t}, J=7.3 \mathrm{~Hz}) .2 .67(1 \mathrm{H}, \mathrm{t}, J=8.0 \mathrm{~Hz}), 2.06(1 \mathrm{H}, \mathrm{dt}, \mathrm{t}, J=7.3 \mathrm{and} 8.0 \mathrm{~Hz}) ;{ }^{13} \mathrm{C} \mathrm{NMR}$ $\left(\mathrm{CDCl}_{3}, 75 \mathrm{MHz}\right) \delta 175.8,159.5,125.0,123.9,120.9,111.0,48.3,34.7,18.2$.

4-hydroxy-5-oxo-4-\{3-[(4,5-dibromo- $1 H$-pyrrole-2-carbonyl)-amino]-propyl $\}$-imidazolin-2-ylidene 17: To an acetic acid $(2 \mathrm{~mL})$ solution of $\mathbf{1 3 + 1 4}\left(73 \mathrm{mg}, 0,2 \mathrm{mmol}\right.$.) was added during $1 \mathrm{~h}$ a solution of $\mathrm{Br}_{2}$ in $\mathrm{AcOH}\left(1 \mathrm{~mL}\right.$ of $4.10^{-4}$ mol. $\mathrm{L}^{-1}$ solution, 2 equiv.). The reaction mixture was stirred overnight at room temperature, quenched with saturated $\mathrm{Na}_{2} \mathrm{CO}_{3}$ and extracted with EtOAc. The organic layer was dried over magnesium sulfate. After evaporation of the solvent the residue was treated with a mixture of $\mathrm{CH}_{2} \mathrm{Cl}_{2} / \mathrm{CF}_{3} \mathrm{CO}_{2} \mathrm{H}: 2 \mathrm{~mL} / 1 \mathrm{~mL}$ during $1 \mathrm{~h}$. Evaporation of the solvents gave $81 \mathrm{mg}$ of the pure 17 in $74 \%$ yield.<smiles>NC1=NC(O)(CCCNC(=O)c2cc(Br)c(Br)[nH]2)C(=O)N1</smiles>

17, $\mathrm{CF}_{3} \mathrm{CO}_{2} \mathrm{H}$ : Amorphous solid, ESI-MS: 425.9-423.9-421,9 [M+H] $]^{+}, 407.9-405.9-403.9\left[\mathrm{M}-\mathrm{H}_{2} 0\right]{ }^{1} \mathrm{H} \mathrm{NMR}\left(\mathrm{CD} \mathrm{OD}_{3}, 300 \mathrm{MHz}\right)$ $\delta 6.78(1 \mathrm{H}), 3.32(2 \mathrm{H}, \mathrm{t}, J=7.0 \mathrm{~Hz}), 1,95(2 \mathrm{H}, \mathrm{t}, J=7.6 \mathrm{~Hz}) .1 .72(1 \mathrm{H}, \mathrm{m}), 1.56(1 \mathrm{H}, \mathrm{m}) .{ }^{13} \mathrm{C} \mathrm{NMR}\left(\mathrm{CD}_{3} \mathrm{OD}, 75 \mathrm{MHz}\right) \delta 175.3$, $161.5,158.9,128.4,113.9,105.7,99.6,87.4,39.5,34.2,23.9$.

\section{Discapamide A (2):}

A methanesulfonic acid $(1.5 \mathrm{~mL})$ solution of $17\left(47 \mathrm{mg}, 0.1 \mathrm{mmol}\right.$.) was heated at $80^{\circ} \mathrm{C}$ during 10 min. After cooling at room temperature, ethyl ether was added the reaction mixture. The precipitate was filtered and washed with ether. The resulting residue was purified on alumina gel $\left(\mathrm{CH}_{2} \mathrm{Cl}_{2} / \mathrm{MeOH}: 3 / 1\right)$ to give $25 \mathrm{mg}$ of dispacamide $\mathrm{A}$ (2) in $65 \%$ yield. All spectral data of synthetic 2 were in satisfactory agreement with those reported for the natural material. ${ }^{4}$<smiles>NC1=N/C(=C/CCNC(=O)c2cc(Br)c(Br)[nH]2)C(=O)N1</smiles> 